\title{
Study on Graphene Based Next Generation Flexible Photodetector for Optical Communication
}

\author{
Kanishka Majumder¹, Devesh Barshilia², Subimal Majee ${ }^{2 *}$ \\ ${ }^{1}$ Academy of Technology, Maulana Abul Kalam Azad University of Technology, Kolkata, India \\ ${ }^{2}$ CSIR-Central Electronics Engineering Research Institute (CEERI), Pilani, India \\ Email: *subimal@ceeri.res.in
}

How to cite this paper: Majumder, K., Barshilia, D. and Majee, S. (2018) Study on Graphene Based Next Generation Flexible Photodetector for Optical Communication. Graphene, 7, 9-16.

https://doi.org/10.4236/graphene.2018.72002

Received: March 7, 2018

Accepted: April 13, 2018

Published: April 16, 2018

Copyright (c) 2018 by authors and Scientific Research Publishing Inc. This work is licensed under the Creative Commons Attribution International License (CC BY 4.0).

http://creativecommons.org/licenses/by/4.0/

\section{cc) (i) Open Access}

\begin{abstract}
We report on the efficient photodetection (PD) properties of graphene based $\mathrm{p}$-i-n photodetector, where all the three layers are either single or multilayer graphene sheets. We report the bandwidth and responsivity performance of the device. This simple structure paves the way for the next generation flexible wireless communication systems. A theoretical model is used to study the carrier distribution and current in a graphene based $\mathrm{p}-\mathrm{i}-\mathrm{n}$ photodetector system.
\end{abstract}

\section{Keywords}

Graphene, Photodetector, Flexible Electronics

\section{Introduction}

Photodetectors (PD) play key role in high performance of optoelectronic and photonic systems. P-i-N photodetector is one type of photodetectors which converts optical signal into electrical response. This type of PD usually consists of heavily doped $\mathrm{P}$ and $\mathrm{N}$ regions, separated by an intrinsic layer. Usual $\mathrm{P}-\mathrm{i}-\mathrm{N}$ PD systems consist of semiconductor materials like, Germanium, Indium gallium arsenide, Lead sulphide and Silicon. The next generation flexible and wearable communication systems require efficient photodetector material which is compatible with the flexible fabrication process. In recent years, copious amount of research is going on to fabricate flexible PD systems using various types of flexible materials, like, Tin Monosulfide, $\mathrm{CsPbBr}_{3}$ microcrystals, two-dimensional (2D) layered materials, organic semiconductors and perovskite materials [1] [2] [3]. Among all existing flexible PD materials, graphene based 
PD systems are attractive research topic owing to its unique advantages. Graphene is a zero band gap hexagonal honeycomb carbon atomic layer [4]-[10], which allows absorption in broad range of wavelengths. Along with other unique properties of graphene, this wonder material is extremely flexible and it has huge potential to be used in flexible device fabrication. In the past few years, graphene based photodetectors have been successfully reported [11] [12] [13] [14], they are mostly fabricated on rigid substrates, providing a limitation of its utilization is flexible electronic systems. Thus, though there are vast applications of photodetector mainly in the nano-metric dimensions with unique features, several areas require immediate attention to optimize the engineered properties of such devices. Implementing a useful model for graphene based photodetector is still under research. In this letter, we describe a model for graphene based $p-i-n$ photodetector based on simple concepts, where all the layers of the device is made of graphene sheets allowing its applicability in flexible electronics applications.

\section{Model}

The model is based on the previously reported study on photodetector devices [15] [16] [17] [18] [19]. We consider the graphene based p-i-n photodetector structure, as shown in Figure 1. For practical fabrication process, mechanically exfoliated graphene single layer or multilayer sheets can be utilized. In order to create doped graphene structures, chemical doping of the graphene sheets can be realized. P-type graphene sheets can be realized by intercalating halogen dopants $(\mathrm{Cl}, \mathrm{Br}, \mathrm{I}$ and $\mathrm{F})$, alkali metal based dopants $\left(\mathrm{K}, \mathrm{Li}, \mathrm{Na}\right.$, etc.), acids $\left(\mathrm{HCl}, \mathrm{HNO}_{3}\right.$ and $\mathrm{H}_{2} \mathrm{SO}_{4}$ ) and some organic compounds [9] [20] [21] [22]; whereas, N-type graphene structures are usually created by intercalating metallic compounds like $\mathrm{ZnMg}$ [23].

Here, the light is incident on the P side. The structure consists of a single layer of $\mathrm{N}^{+}$graphene layer, an undoped multi-layer graphene with thickness $I$ and finally a single layer graphene $\mathrm{P}^{+}$layer. The nominal $\mathrm{N}^{+}$and $\mathrm{P}^{+}$region doping is taken of the order of $1 \times 10^{12} \mathrm{~cm}^{-2}$, which is practically reliable value [24].

In the theoretical model, we have to consider the effect of photogenerated carriers in the intrinsic layer as because the widths of the three regions are comparable to each other. Thus, the current continuity equations in the depletion layer is given by [15]

$$
\frac{\partial n(x, t)}{\partial t}-v_{n}\left\{\frac{\partial n(x, t)}{\partial x}\right\}=g
$$

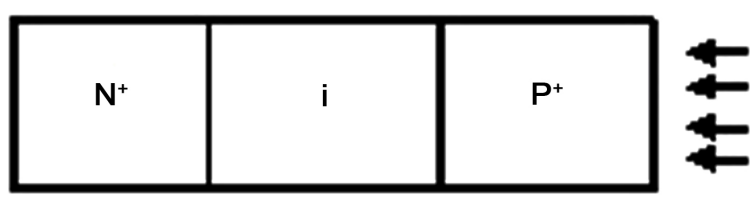

Figure 1. Schematic structure of a graphene based p-i-n photodetector. 


$$
\frac{\partial p(x, t)}{\partial t}+v_{p}\left\{\frac{\partial p(x, t)}{\partial x}\right\}=g
$$

where, $g$ is the photo carrier generation rate, $v$ is the velocity, $n$ and $p$ denote the electron and hole, respectively. Incident optical powers, absorption coefficient of graphene at the operating wavelength, reflectivity of the graphene surface are some of the important parameters which control the generation rate of the photo carriers.

Carrier distribution $N(x, j \omega)$ for electrons and $P(x, j \omega)$ for holes in the depletion region (in frequency domain) are calculated by solving the above current continuity equations simultaneously. In general, all the uppercase variables are used to indicate the Laplace transform of the corresponding lowercase variables. The entire depletion region is subdivided into equal energy spacing $(\Delta x)$ for calculation. Along its path of motion, each carrier represents a specific position and energy state in the depletion region of the device. For this reason, each carrier is specifically represented as a function of two indices: one position index $(i)$ and one energy index $(j)$. So, we substitute $N(x, j \omega)$ by $N(i, j, j \omega)$ and $P(x, j \omega)$ by $P(i, j, j \omega)$.

To obtain the photo-current density, the carrier distribution $N(i, j, j \omega)$ and $P(i, j, j \omega)$ are multiplied by equal energy spacing $(\Delta x)$ and then summed for all $i$ and $j$. The current density Jof the device is obtained using Equation (3)

$$
J=\frac{q}{L} \sum_{i}\left[\sum_{j}\left\{N(i, j, j \omega) v_{n}(i)+P(i, j, j \omega) v_{p}(i)\right\}\right] \Delta x(i)
$$

where, $L, q$ and $v$ are the length of the PD, electronic charge and the carrier velocity respectively. We consider here that the carrier velocity is only function of the position. Suffix $n$ and $p$ is used for electrons and for holes respectively.

\section{Results and Discussions}

The material parameters for the graphene layer have been taken from the literature [11] [12] [13] [14] [24]. Using those parameters in this present model, 3-dB bandwidth, frequency response and responsivity of the device have been calculated. The calculated values are justified by the performance of the fabricated graphene based PD devices as described in literature [11] [12] [13] [14].

Figure 2 shows the $3-\mathrm{dB}$ bandwidth variation i-layer thickness, where $\mathrm{p}$ and $\mathrm{n}$ layer consist of single layer graphene sheets (thickness $\sim 1 \mathrm{~nm}$ ). Areas of the PD devices are also varied in this case. Maximum bandwidth $(\sim 5 \mathrm{GHz})$ is obtained for the multilayer graphene i-layer $(5 \mathrm{~nm})$. Effect of device dimension on bandwidth can also be observed from the plot, where smaller sized devices have better bandwidth compare to the larger sized devices. Keeping i-layer thickness constant at $5 \mathrm{~nm}$ where maximum bandwidth is obtained, the $\mathrm{P}^{+}$and $\mathrm{N}^{+}$layer thicknesses are varied from 1 to $5 \mathrm{~nm}$ and area of the device is also varied, as seen from Figure 3. There is no significant variation of the 3-dB bandwidth when the $\mathrm{P}^{+}$and $\mathrm{N}^{+}$layer thicknesses are changed, which concludes that the 


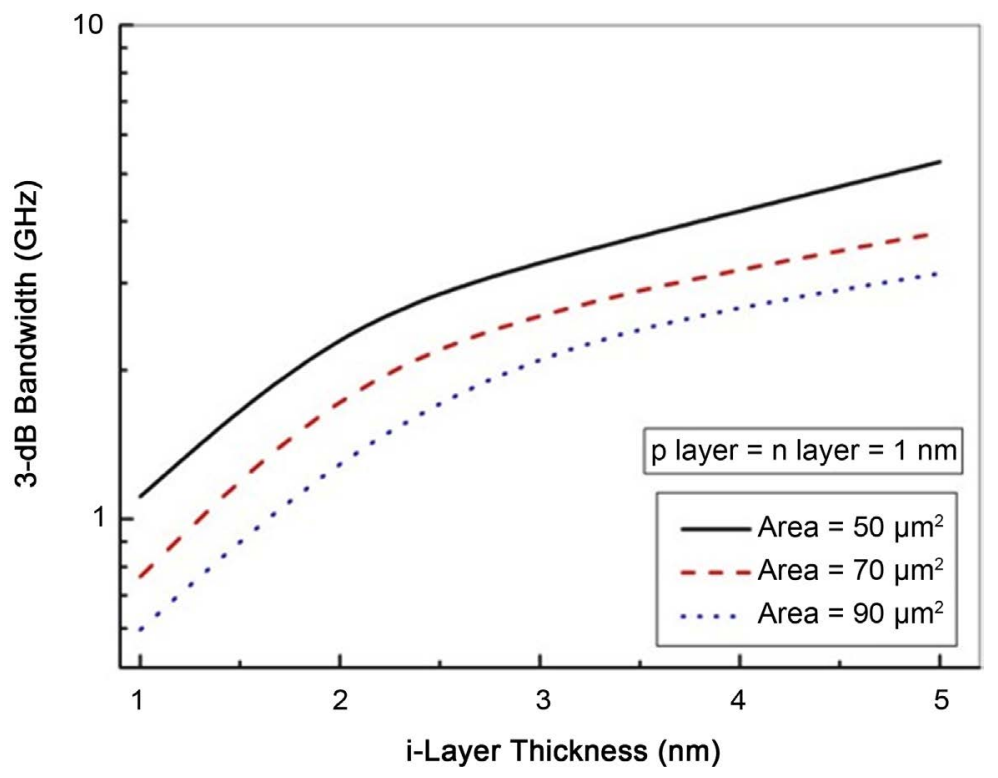

Figure 2. Variation of bandwidth with i-layer thickness keeping $\mathrm{N}^{+}$and $\mathrm{P}^{+}$-layer thicknesses fixed at $1 \mathrm{~nm}$.

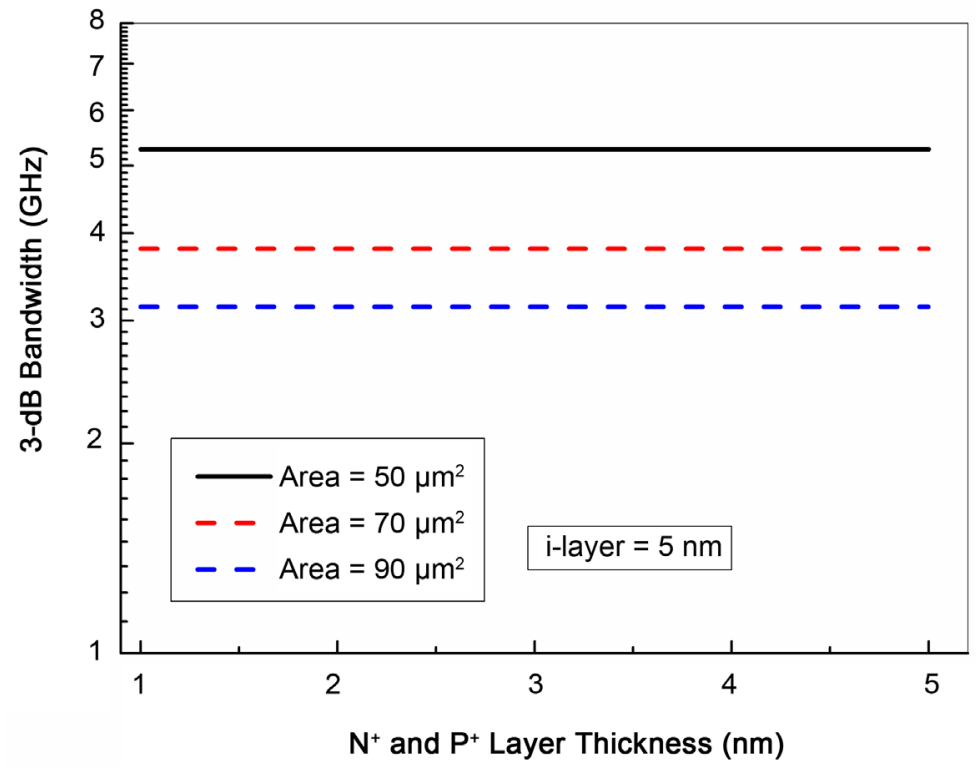

Figure 3. Variation of bandwidth with $\mathrm{N}^{+}$and $\mathrm{P}^{+}$-layer thicknesses keeping i-layer thickness fixed at $5 \mathrm{~nm}$.

bandwidth of the device is dependent on the intrinsic layer thickness. This conclusion facilitates the applicability of the model in real fabrication of the device, since producing multilayer graphene sheets is comparatively easier than single layer graphene sheets.

Figure 4 shows the frequency response, keeping device area fixed at $50 \mu \mathrm{m}^{2}$, which gave rise to the maximum 3-dB bandwidth as observed from Figure 2 and Figure 3. The i-layer thickness is varied from single layer graphene to few layers of graphene. Again we observe the multilayer graphene i-layer gives rise to better 
response. This is likely due to the fact that multilayer graphene sheets have poor electrical conductivity as compared to the single layer graphene sheets, giving rise to better performance of the intrinsic layer.

Keeping i-layer thickness as $5 \mathrm{~nm}$ and device area as $50 \mu \mathrm{m}^{2}$, the responsivity of the system has been investigated, where $\mathrm{p}$ and $\mathrm{n}$ layer thicknesses are kept fixed at $1 \mathrm{~nm}$. Figure 5 shows the change of responsivity of the PD system with

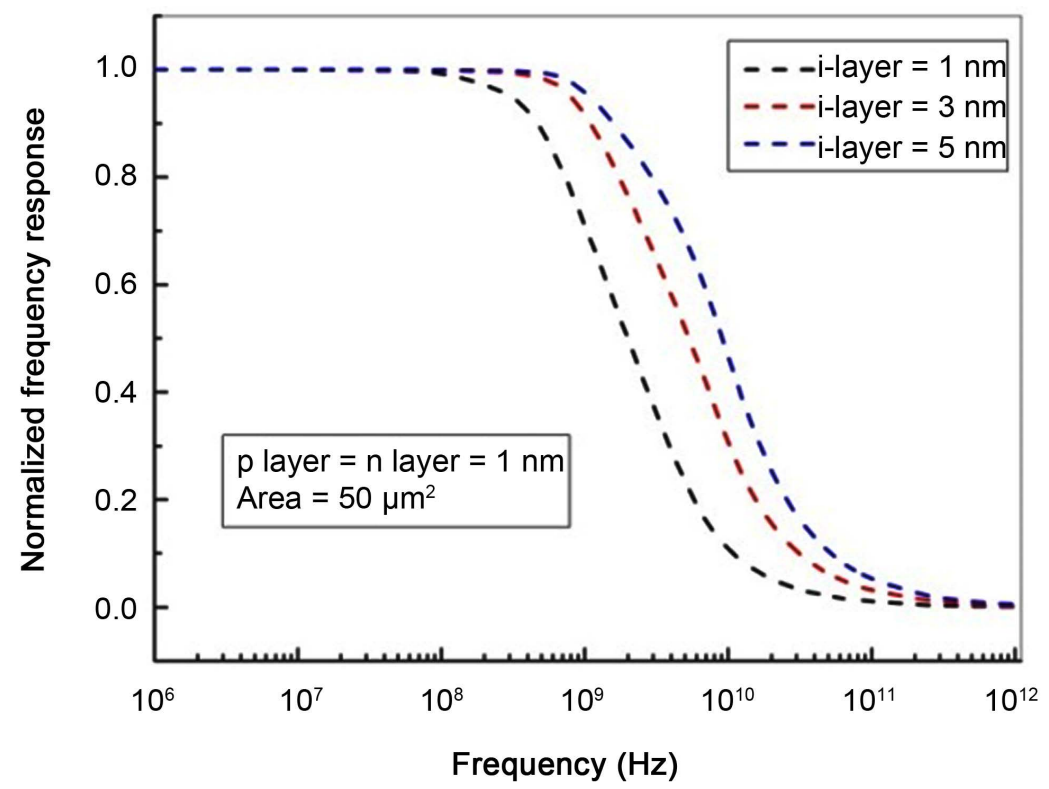

Figure 4. Normalized frequency response with i-layer thickness.

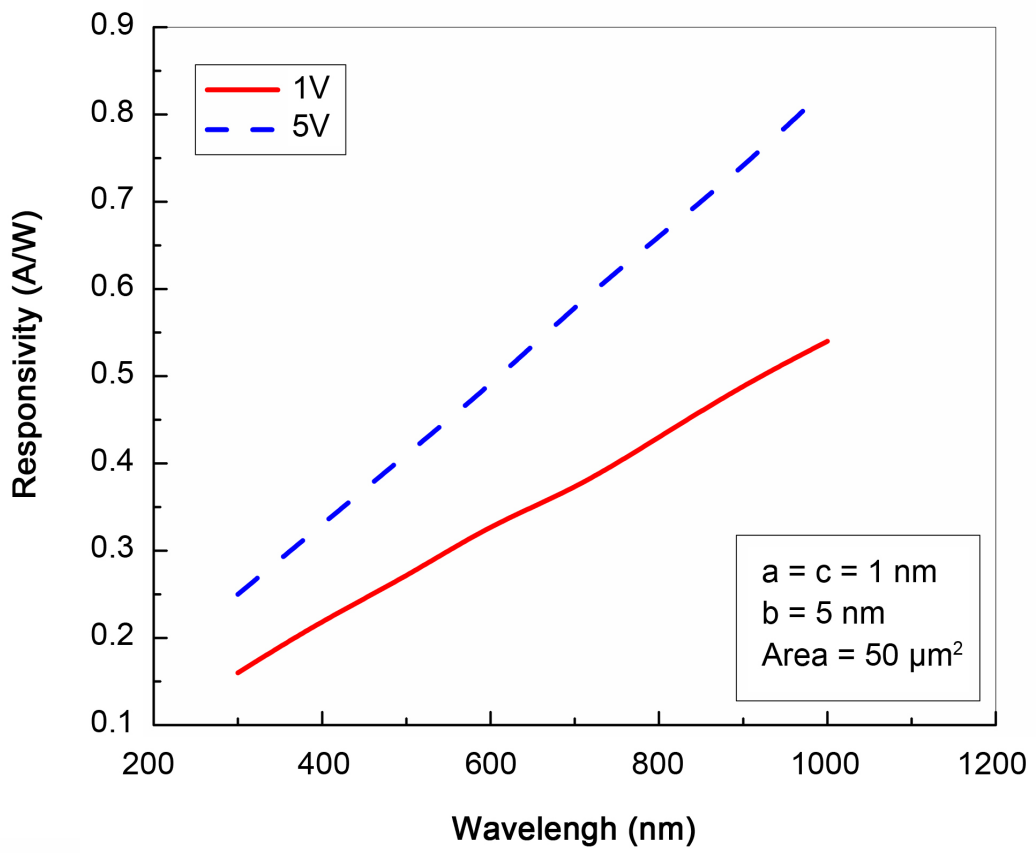

Figure 5. Responsivity is plotted as a function of wavelength for $5 \mathrm{~nm}$ i-layer (b) thickness in a Graphene-Graphene-Graphene $\mathrm{n}^{+}-\mathrm{i}-\mathrm{p}^{+}$Photodiode (PD). $\mathrm{n}^{+}(\mathrm{a})$ and $\mathrm{p}^{+}(\mathrm{c})$ Graphene layer thicknesses are taken to be $1 \mathrm{~nm}$. 


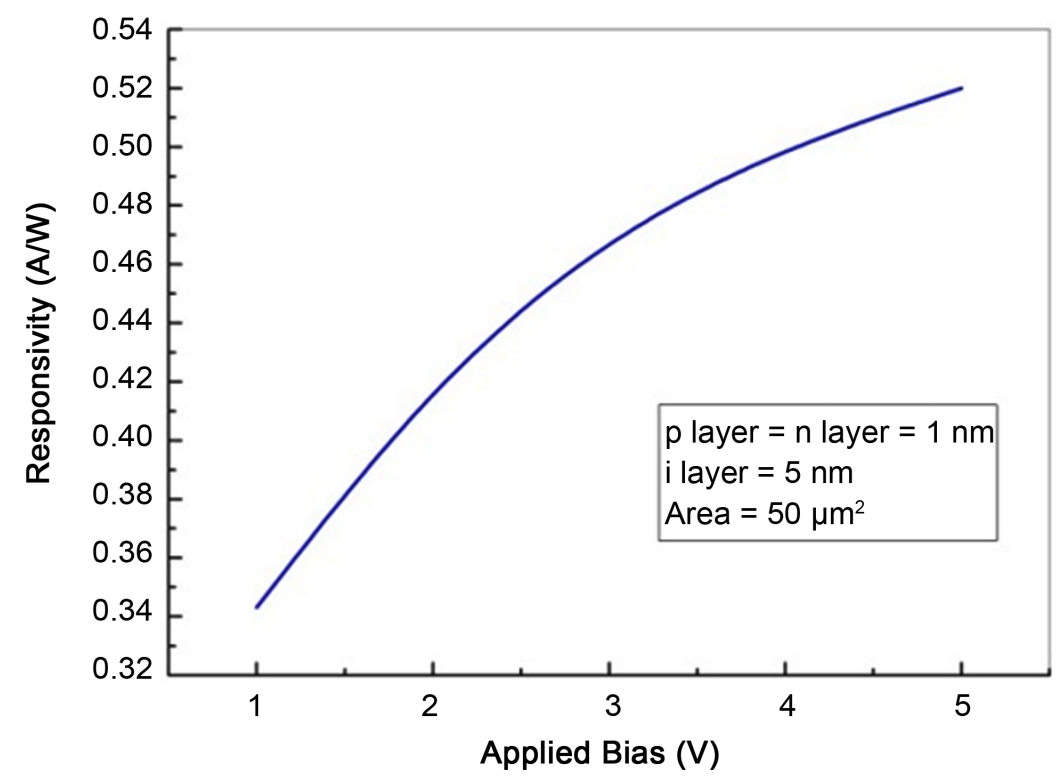

Figure 6. Responsivity is plotted as a function of bias at $633 \mathrm{~nm}$ for $5 \mathrm{~nm}$ i-layer (b) thickness in a Graphene-Graphene-Graphene $\mathrm{n}^{+}-\mathrm{i}-\mathrm{p}^{+}$Photodiode (PD). $\mathrm{n}^{+}(\mathrm{a})$ and $\mathrm{p}^{+}(\mathrm{c})$ Graphene layer thicknesses are taken to be $1 \mathrm{~nm}$.

Table 1. Performance of $\mathrm{p}-\mathrm{i}-\mathrm{n}$ Photodetector (PD) based on Graphene: $\mathrm{p}=\mathrm{n}=1 \mathrm{~nm}, \mathrm{i}=$ $5 \mathrm{~nm}, \mathrm{~V}=1.5 \mathrm{~V}$.

\begin{tabular}{ccccc}
\hline Area $\left(\mu \mathrm{m}^{2}\right)$ & $\begin{array}{c}\text { Bandwidth } \\
(\mathrm{GHz})\end{array}$ & $\begin{array}{c}\text { Responsivity } \\
(\mathrm{A} / \mathrm{W})\end{array}$ & Rise Time (Sec) & EQE (\%) \\
\hline 50 & 5.28 & 0.34 & $0.066 \times 10^{-9}$ & 67.23 \\
\hline
\end{tabular}

wavelength, which shows a linear response of the device for different applied bias voltages. We also investigate the responsivity of the $\mathrm{PD}$ at $633 \mathrm{~nm}$ wavelength, as a function of applied bias which is comparable with the reported values in the literature [14] as shown in Figure 6. Table 1 shows the performance of $p-i-n$ Photodetector (PD) based on the optimized parameters discussed in this paper.

\section{Conclusion}

We have designed an effective graphene based flexible PD system whose performance matches well with the experimental values in the literature. The PD system can be effectively used in the next generation communication systems. Further investigations are under progress to fabricate graphene based PD devices on flexible polymer substrates.

\section{Acknowledgements}

The authors would like to thank Prof. S. Chaudhury, Director CEERI-Pilani and Prof. D. Bhattacharya, Director AOT for their support.

\section{References}

[1] Kumar, G.M., Fu, X., Ilanchezhiyan, P., Yuldashev, S.U., Lee, D.J., Cho, H.D. 
and Kang, T.W. (2017) Highly Sensitive Flexible Photodetectors Based on Self-Assembled Tin Monosulfide Nanoflakes with Graphene Electrodes. ACS Applied Materials \& Interfaces, 9, 32142-32150.

https://doi.org/10.1021/acsami.7b09959

[2] Cao, F., Yu, D.J., Li, X.M., Zhu, Y., Sun, Z.G., Shen, Y.L., Wu, Y., Wei, Y. and Zeng, H.B. (2017) Highly Stable and Flexible Photodetector Arrays Based on Low Dimensional $\mathrm{CsPbBr} 3$ Microcrystals and On-Paper Pencil-Drawn Electrodes. Journal of Materials Chemistry C, 5, 7441-7445. https://doi.org/10.1039/C7TC02108A

[3] Xie, C. and Yan, F. (2017) Flexible Photodetectors Based on Novel Functional Materials. Small, 13, 1701822. https://doi.org/10.1002/smll.201701822

[4] Bolotin, K.I., Sikes, K.J., Jiang, Z., Klima, M., Fudenberg, G., Hone, J., Kim, P. and Stormer, H.L. (2008) Ultrahigh Electron Mobility in Suspended Graphene. Solid State Communications, 146, 351. https://doi.org/10.1016/j.ssc.2008.02.024

[5] Geim, A.K. and Novoselov, K.S. (2007) The Rise of Graphene. Nature Materials, 6, 183-191. https://doi.org/10.1038/nmat1849

[6] Castro Neto, A.H., Guinea, F., Peres, N.M.R., Novoselov, K.S. and Geim, A.K. (2009) The Electronic Properties of Graphene. Reviews of Modern Physics, 81, 109. https://doi.org/10.1103/RevModPhys.81.109

[7] Majee, S., Song, M., Zhang, S.-L. and Zhang, Z.-B. (2016) Scalable Inkjet Printing of Shear-Exfoliated Graphene Transparent Conductive Films. Carbon, 102, 51-57.

[8] Majee, S., Banerjee, D., Liu, X., Zhang, S.-L. and Zhang, Z.-B. (2017) Efficient and Thermally Stable Iodine Doping of Stacked Graphene Nano-Platelets. Carbon, 117, 240-245.

[9] Majee, S., Liu, C., Wu, B., Zhang, S.-L. and Zhang, Z.-B. (2016) Ink-Jet Printed Highly Conductive and Reliable Pristine Graphene Patterns Achieved with Water-Based Ink and Aqueous Doping Processing. Carbon, 114, 77-83. https://doi.org/10.1016/j.carbon.2016.12.003

[10] Miao, F., Majee, S., Song, M., Zhao, J., Zhang, S.-L. and Zhang, Z.-B. (2016) Inkjet Printing of Electrochemically-Exfoliated Graphene Nano-Platelets. Synthetic Metals, 220, 318-322.

[11] Gabor, N.M., Song, J.C., Ma, Q., Nair, N.L., Taychatanapat, T., Watanabe, K., Taniquchi, T., Levitov, L.S. and Jarillo-Herrero, P. (2011) Hot Carrier-Assisted Intrinsic Photoresponse in Graphene. Science, 334, 648-652.

https://doi.org/10.1126/science.1211384

[12] Lemme, M.C., Koppens, F.H.L., Falk, A.L., Rudner, M.S., Park, H., Levitov, L.S. and Marcus, C.M. (2011) Gate-Activated Photoresponse in a Graphene p-n Junction. Nano Letters, 11, 4134-4137. https://doi.org/10.1021/nl2019068

[13] Freitag, M., Low, T. and Avouris, P. (2013) Increased Responsivity of Suspended Graphene Photodetectors. Nano Letters, 13, 1644-1648.

https://doi.org/10.1021/nl4001037

[14] An, X., Liu, F., Jung, Y.J. and Kar, S. (2013) Tunable Graphene-Silicon Heterojunctions for Ultrasensitive Photodetection. Nano Letters, 13, 909-916.

https://doi.org/10.1021/nl303682j

[15] Majumder, K. and Das, N.R. (2012) Modeling the Gain and Bandwidth of Submicron Active Layer n+-i-p+ Avalanche Photodiode. Proceedings of the 16th International Workshop on Physics of Semiconductor Devices, 854-902. https://doi.org/10.1117/12.925141

[16] Majumder, K. and Das, N.R. (2014) Effect of Ge-Composition on the Gain of a Thin 
Layer Si1-yGey Avalanche Photodiode. In: Jain, V. and Verma, A., Eds., Physics of Semiconductor Devices, Environmental Science and Engineering, Springer, Cham, 219-221. https://doi.org/10.1007/978-3-319-03002-9_54

[17] Das, N.R. and Deen, M.J. (2002) A New Model for Avalanche Build-Up of Carriers in a SAGCM Avalanche Photodiode. IEEE Transactions on Electron Devices, 49, 2362-2366.

[18] Majumder, K. and Das, N.R. (2013) Effect of Ge-Composition on the Frequency Response of a Thin Layer Sil-yGey Avalanche Photodiode, Microwave and Photonics (ICMAP).

[19] Das, N.R. and Deen, M.J. (2002) On the Frequency Response of a Resonant-Cavity-Enhanced Separate Absorption, Grading, Charge, and Multiplication Avalanche Photodiode. Journal of Applied Physics, 92, 7133-7145.

https://doi.org/10.1063/1.1521786

[20] Kim, H., Renault, O., Tyurnina, A., Simonato, J.-P., Rouchon, D., Mariolle, D., et al. (2014) Doping Efficiency of Single and Randomly Stacked Bilayer Graphene by Iodine Adsorption. Applied Physics Letters, 105, Article ID: 011605. https://doi.org/10.1063/1.4889747

[21] Zhao, Y., Wei, J., Vajtai, R., Ajayan, P.M. and Barrera, E.V. (2011) Iodine Doped Carbon Nanotubes Cables Exceeding Specific Electrical Conductivity of Metals. Scientific Reports, 1, 83. https://doi.org/10.1038/srep00083

[22] Zhan, Y., Zhang, B., Cao, L., Wu, X., Lin, Z., Yu, X., Zhang, X., Zeng, D., Xie, F., Zhang, W., Chen, J. and Meng, H. (2015) Iodine Doped Graphene as Anode Material for Lithium Ion Battery. Carbon, 94, 1. https://doi.org/10.1016/j.carbon.2015.06.039

[23] Meng, X., Tongay, S., Kang, J., Chen, Z., Wu, F., Li, S.S., Xia, J.B., Li, J. and Wu, J. (2013) Stable p- and n-Type Doping of Few-Layer Graphene/Graphite. Carbon, 57, 507-514. https://doi.org/10.1016/j.carbon.2013.02.028

[24] Lee, J.K., Sung, H., Jang, M.S., Yoon, H. and Choi, M. (2015) Reliable Doping and Carrier Concentration Control in Graphene by Aerosol-Derived Metal Nanoparticles. Journal of Materials Chemistry C, 3, 8294-8299.

https://doi.org/10.1039/C5TC01443C 